\title{
(G) Standardised criteria for OPEN ACCESS ECG interpretation in athletes: a practical tool
}

\author{
Jonathan A Drezner
}

Cardiovascular-related sudden death is the leading cause of mortality in athletes during sport. ${ }^{1}$ The majority of disorders associated with increased risk of sudden cardiac death (SCD), such as cardiomyopathies and primary electrical diseases, are suggested by abnormal findings present on an ECG. ECG interpretation in athletes requires careful analysis to properly distinguish physiological changes related to athlete's heart from findings suggestive of an underlying pathological cardiac condition. Whether used for the diagnostic evaluation of cardiovascular-related symptoms, a family history of inheritable cardiac disease or SCD, or for screening of asymptomatic athletes, ECG interpretation is an important skill for physicians involved in the cardiovascular care of athletes.

The purpose of this short report is to guide the clinician on the use of a two-page ECG criteria tool (Appendix,

Department of Family Medicine, University of Washington, Seattle, Washington, USA

Correspondence to Professor Jonathan A Drezner, Department of Family Medicine, University of Washington, P.O. Box 354410, Seattle, WA 98195, USA; jdrezner@uw.edu pages $\mathbf{i 7 - i 8 ) . ~ T h e ~ s c i e n c e ~ u n d e r p i n n i n g ~}$ this instrument has been reported ${ }^{2}$ and demonstrates that providing standardised criteria to assist ECG interpretation in athletes significantly improves accuracy to distinguish normal from abnormal findings across physician specialties, even in physicians with little or no experience. The first page of the criteria tool consists of two tables. Table 1 lists each individual ECG criterion that should be considered abnormal, unrelated to athletic training, and warrants additional investigation for disorders predisposing to SCD. Table 2 lists the normal and physiological ECG changes commonly found in trained athletes that should not trigger additional testing. The second page of the tool (Appendix, page i8) displays several examples of uncommon ECG patterns.

\section{CLASSIFYING ECGs AS NORMAL OR ABNORMAL}

The goal of ECG interpretation in athletes is to classify the ECG as: (1) 'normal'-no further evaluation needed or (2) 'abnormal'-further evaluation needed. Normal ECGs include common, training-related findings in athletes such as high ORS amplitude meeting voltage criteria for left ventricular hypertrophy, early repolarisation, sinus bradycardia, sinus arrhythmia and $1^{\circ}$ atrioventricular block. Abnormal findings are unrelated to regular training and also found in underlying pathological cardiac conditions. These include findings suggestive of cardiomyopathy such as T-wave inversion, ST depression, pathological Q-waves, left axis deviation and conduction delays and findings suggestive or diagnostic of primary electrical diseases such as long QT syndrome and WolffParkinson-White syndrome.

The evaluation of abnormal ECG findings is ideally performed in consultation with a specialist with experience in athlete's heart and disorders associated with SCD in athletes. However, even if properly interpreted, ECG will not detect all conditions at risk for SCD. Further diagnostic testing often requires advanced cardiac imaging (echocardiography or cardiac magnetic resonance), ambulatory or exercise ECG monitoring, and less commonly genetic testing or electrophysiological study. Physicians may modify the ECG criteria or diagnostic testing based on past experience, practice setting or the presence or absence of concerning symptoms or family history.

\section{AN IMPORTANT STEP BUT WHAT IS NEXT?}

This practical tool (which may be reproduced unchanged with citation to the $B J S M$ pages $\mathbf{i 7 - i 8 )}$ represents only a starting point to improve ECG interpretation in athletes. Physicians also may benefit by reviewing published guidelines outlining modern criteria for ECG interpretation in athletes. ${ }^{3-5}$ In addition, an international group of experts convened 
in Seattle in February 2012 to refine ECG interpretation criteria and develop an online training module for physicians around the world to gain a common foundation in ECG interpretation in athletes. This free online training course, cosponsored by the American Medical Society for Sports Medicine and FIFA, will be hosted by BMJ Learning and will launch early in 2013. Until then, use of this instrument can provide physicians standardised criteria to assist them in interpreting an athlete's ECG.
- Additional data are published online only. To view this file please visit the journal online (http://dx.doi.org/ 10.1136/bjsports-2012-091703)

Competing interests None.

Provenance and peer review Commissioned; internally peer reviewed.

Accepted 21 August 2012

Br J Sports Med 2012:46:i6-i8.

doi:10.1136/bjsports-2012-091703

\section{REFERENCES}

1. Harmon KG, Asif IM, Klossner D, et al. Incidence of sudden cardiac death in national collegiate athletic association athletes. Circulation 2011;123:1594-600.
2. Drezner JA, Asif IM, Owens DS, et al. Accuracy of ECG interpretation in competitive athletes: the impact of using standised ECG criteria. Br J Sports Med 2012;46:335-40.

3. Williams ES, Owens DS, Drezner JA, et al. Electrocardiogram interpretation in the athlete. Herzschrittmacherther Elektrophysiol 2012;23:65-71.

4. Corrado D, Pelliccia A, Heidbuchel $\mathrm{H}$, et al. Recommendations for interpretation of 12-lead electrocardiogram in the athlete. Eur Heart $J$ 2010;31:243-59.

5. Uberoi A, Stein R, Perez MV, et al. Interpretation of the electrocardiogram of young athletes. Circulation 2011;124:746-57

\section{APPENDIX ECG INTERPRETATION IN ATHLETES}

Table 1 Abnormal ECG criteria in Athletes Any abnormal finding is considered training-unrelated and suggests the possibility of underlying pathological cardiac disease, requiring further diagnostic work-up.

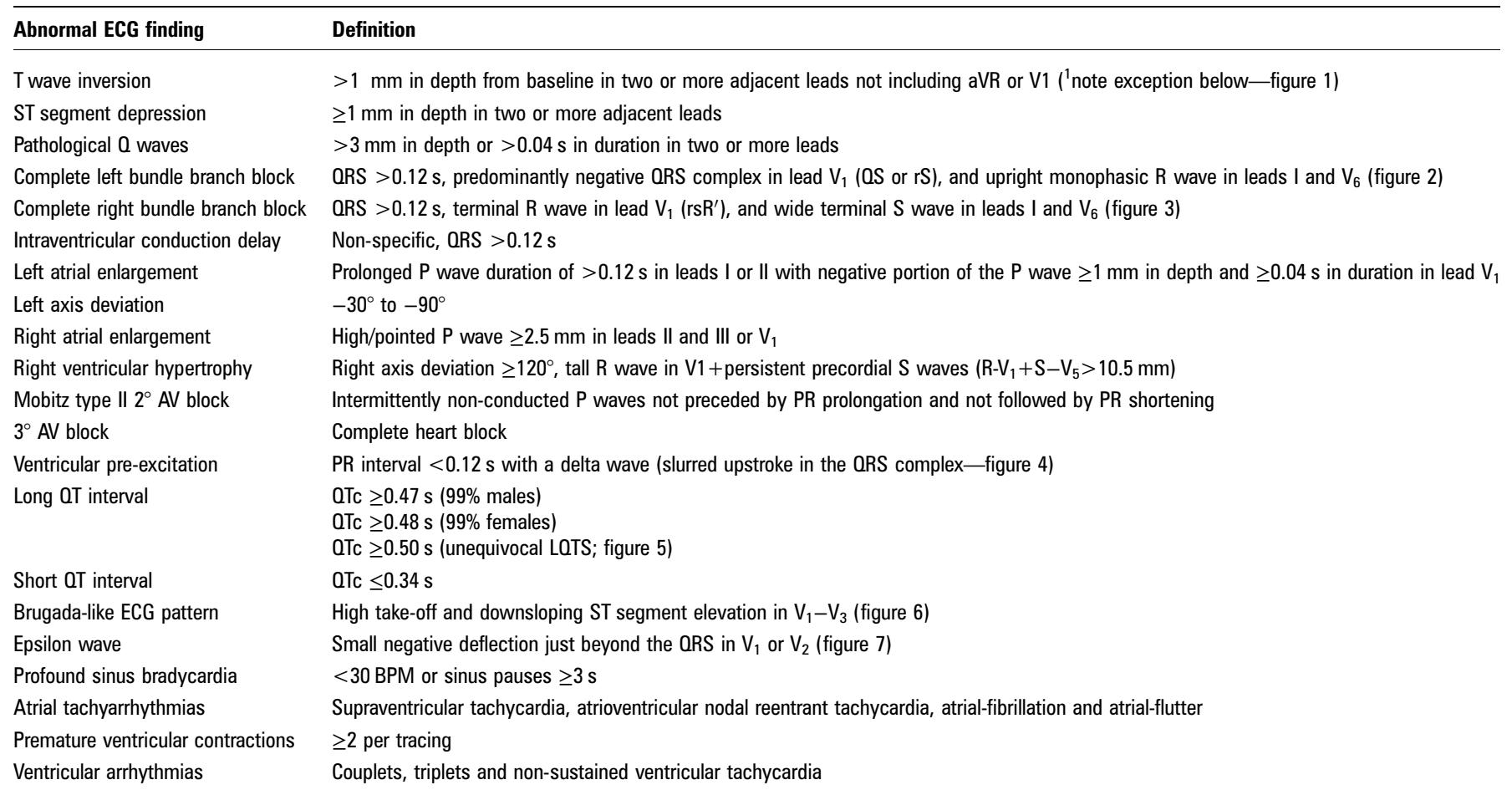

${ }^{1}$ Note: Exception to T wave inversion: elevated ST-segment with an upward ('domed') convexity, followed by a negative T-wave in $\mathrm{V}_{2}-\mathrm{V}_{4}$ is a common pattern of early repolarisation seen in athletes of African-Caribbean descent and should be considered normal (figure 1). This should not to be confused with the downsloping ST segment elevation in $V_{1}-V_{3}$ found in a Brugada-like ECG pattern which is abnormal (figure 6). $\mathrm{AV}$, atrioventricular.

Table 2 Common ECG findings in athletes Training-related ECG alterations are common, physiological adaptations to regular exercise and are considered normal variants in athletes.

\begin{tabular}{lll}
\hline Sinus bradycardia & First degree AV block & Early repolarisation \\
\hline Sinus arrhythmia & Incomplete RBBB & Isolated QRS voltage criteria for left ventricular hypertrophy ${ }^{2}$
\end{tabular}

${ }^{2}$ Note: Isolated increases in QRS amplitude are common in trained athletes. However, QRS voltage criteria for LVH+any non-voltage criteria for LVH (such as atrial enlargement, left axis deviation, a 'strain' pattern of repolarisation, ST-segment depression, T-wave inversion or pathological 0 waves) is abnormal and requires further evaluation. LVH, left ventricular hypertrophy; RBBB, right bundle branch block. 


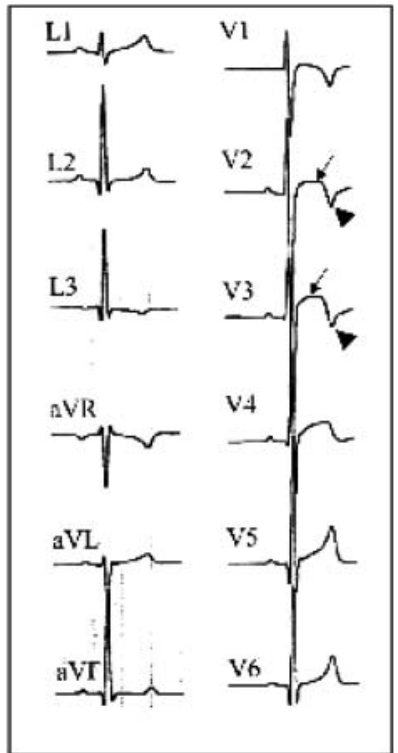

Figure 1 Normal variant of T wave inversion in athletes of African-Caribbean descent.

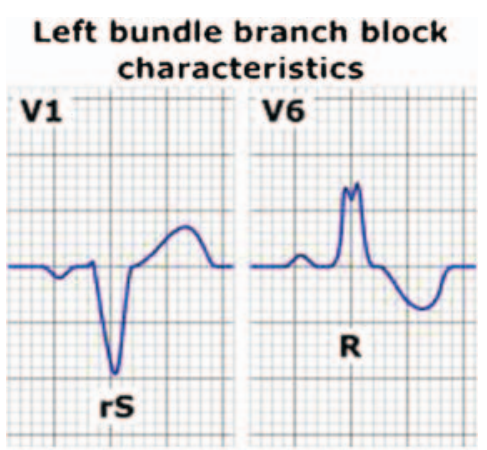

Figure 2 Left bundle branch block: QRS $>0.12 \mathrm{~s}$, predominantly negative QRS complex in lead $V_{1}$ (QS or $r S$ ), and upright monophasic $R$ wave in leads $I$ and $V_{6}$.

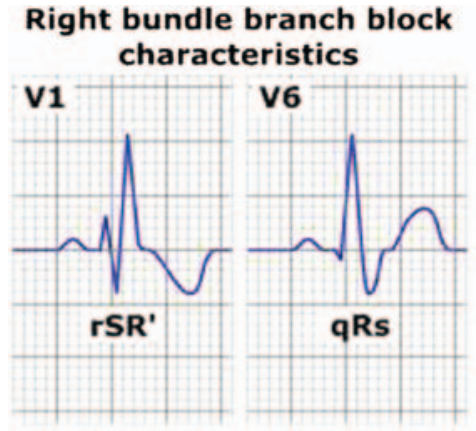

Figure 3 Right bundle branch block: QRS $>0.12 \mathrm{~s}$, terminal $\mathrm{R}$ wave in lead $\mathrm{V}_{1}\left(\mathrm{rs}^{\prime}\right)$, and wide terminal $S$ wave in leads $I$ and $V_{6}$.

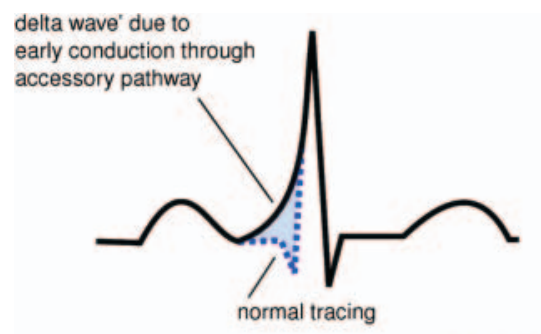

Figure 4 Delta wave: suggestive of ventricular pre-excitation; PR interval $<0.12 \mathrm{~s}$ with or without a delta wave (slurred upstroke in the QRS complex).

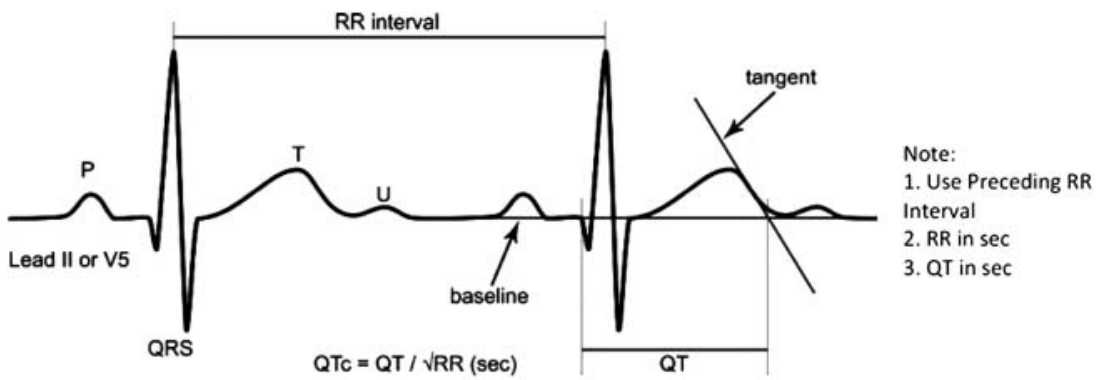

Figure 5 OTc interval: LONG OT: OTc $\geq 0.47 \mathrm{~s}$ ( $99 \%$ males) or OTC $\geq 0.48 \mathrm{~s}$ ( $99 \%$ females). (OTC $\geq 0.50$ s (unequivocal LOTS)).

Figure 6 Brugada ECG: high take-off and downsloping ST segment elevation in $\mathrm{V}_{1}-\mathrm{V}_{3}$.
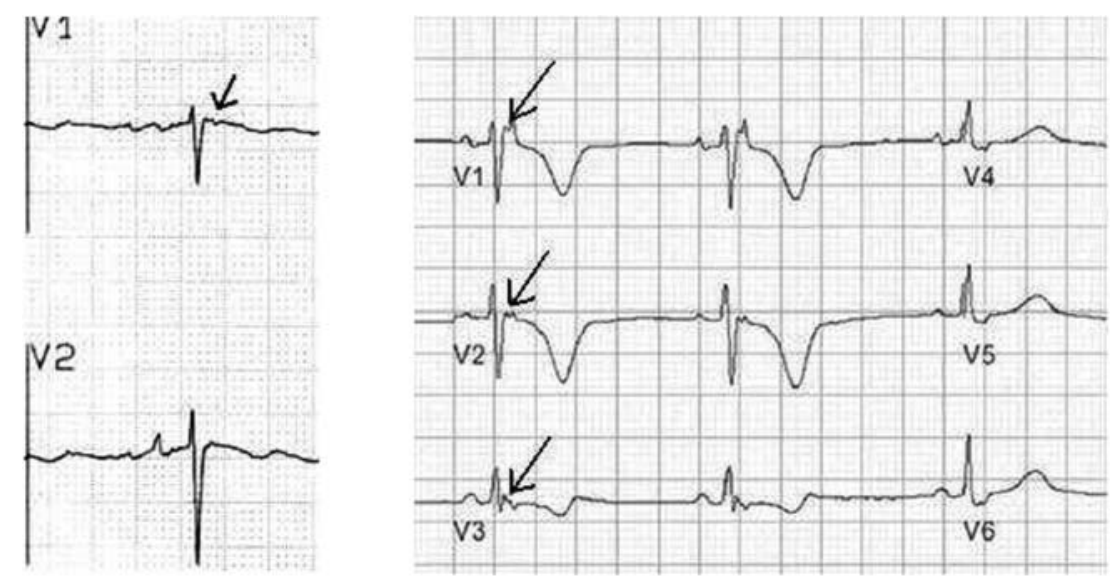

Figure 7 Epsilon wave: small negative deflection just beyond the $\mathrm{QRS}$ in $\mathrm{V}_{1}$ or $\mathrm{V}_{2}$.

[ECG criteria based on: Corrado D, Pelliccia A, Heidbuchel H, et al. Recommendations for interpretation of 12-lead electrocardiogram in the athlete. Eur Heart J 2010;31:243-59] 